\title{
Current Understanding of Ablative Radiation Therapy in Hepatocellular Carcinoma
}

\author{
Ashwathy S Mathew' \\ Laura A Dawson ${ }^{2,3}$ \\ 'Department of Radiation Oncology, \\ Apollo Proton Cancer Centre, Chennai, \\ Tamil Nadu, India; ${ }^{2}$ Radiation Medicine \\ Program, Princess Margaret Cancer \\ Centre, Toronto, Ontario, Canada; \\ ${ }^{3}$ Department of Radiation Oncology, \\ University of Toronto, Toronto, Ontario, \\ Canada
}

\begin{abstract}
The role of ablative stereotactic body radiotherapy (SBRT) in hepatocellular carcinoma (HCC) has been evolving over the last few decades. SBRT has mostly been used in early stages of HCC, including few ( $\leq 3$ in number) tumors, small tumours $(<3 \mathrm{~cm}$ in size), as well as larger tumours which are ineligible for other ablative modalities, mostly without vascular invasion. In early stage HCC, SBRT is used as a definitive treatment with curative intent or with intent to bridge to liver transplant. Retrospective and prospective institutional series document a high rate of local control (68-95\% at 3 years) following SBRT. This coupled with a low risk of toxicity makes this non-invasive ablative treatment an attractive option for patients who are ineligible for other ablative treatments. Small randomized studies of ablative radiation have also shown non-inferiority of radiation as compared to radiofrequency ablation (RFA). Currently, SBRT is widely available as a safe and effective liver directed therapy, although there is a need for more studies providing higher level evidence. This review gives a brief overview of SBRT and the evidence for its use in HCC patients with ablative intent.
\end{abstract}

Keywords: hepatocellular carcinoma, radiotherapy, stereotactic body radiotherapy, SBRT, stereotactic ablative radiotherapy, SABR

\section{Introduction}

Stereotactic body radiotherapy (SBRT) was first used to treat patients with hepatocellular carcinoma (HCC) almost 20 years ago, but still there is a limited amount of high level evidence for its efficacy. ${ }^{1,2}$ Hepatocellular carcinoma (HCC) is the 7 th most common cancer in the world in terms of incidence and the 3rd highest in terms of mortality. ${ }^{3}$ Even though only $15-20 \%$ of HCC patients are eligible for other ablative liver directed treatments (resection, liver transplant, radiofrequency ablation), radiation therapy (SBRT, protons or conformal radiotherapy) is often not included as a treatment option in the BCLC classification due to the lack of highpower randomized trials. The Modified Barcelona Clinic Liver Cancer (BCLC) classification system classifies HCC into Stage 0, A, B, C and D on the basis of various prognostic factors including tumor size, tumor number, liver function and the patient's performance status. ${ }^{4}$ The definition of BCLC A, or "early stage HCC", as per the Modified BCLC staging system includes up to 3 tumor nodules $\leq 3 \mathrm{~cm}$ in size in patients with preserved liver function (Child Pugh A without ascites) and excellent performance status (PS 0), but other definitions of early stage HCC are also used clinically. For example, the AJCC/UICC TNM classification system includes tumors up to $5 \mathrm{~cm}$ in size as Stage I 
\& II disease. ${ }^{5}$ This variability in the definitions of "early stage" HCC is reflected in the heterogeneity of patients included in the various studies reporting outcomes of SBRT. It is generally agreed that small $(<3 \mathrm{~cm}$ in maximum dimension) and limited tumor number $(<5$ in number) confined to the liver with no vascular invasion or extrahepatic spread mostly fall into the category of early HCC. The BCLC classification that gives a broad purview of first line treatment options on the basis of currently available evidence, has been the backbone of HCC treatment guidelines from organizations like the European Association for the Study of Liver Diseases (EASLD) and the American Association for the Study of Liver Diseases (AASLD), and for the purpose of this review, the BCLC definition of early stage HCC will be used, although there is also increasing evidence for SBRT for more advanced stage disease. At the same time, we acknowledge the limitations of the currently available data and emphasize the need to include more homogenous patient populations in future studies, so that the evidence base for SBRT in this patient population is not subject to the lack of power inherent in heterogenous cohorts. Uniform use of a single criterion/definition across the scientific community in future planned studies, will help to easily compare results from different institutions.

Radiation therapy was first used in the treatment of primary liver cancer in the 1980s, although generally as a palliative treatment targeting the whole liver. Early reports quickly recognized the possibility of radiation induced liver disease. The advent of 3-dimensional conformal techniques greatly improved the ability to deliver tumoricidal doses to a part of the liver while sparing the remaining normal functional liver. Three-dimensional conformal radiotherapy (3-DCRT) was developed in the 1990s when newer technology became available in the form of computerized treatment planning systems, used to calculate radiation doses in a 3-dimensional model of the patient body acquired using a CT scan, and deliver ionizing radiation therapy using multi-leaf collimators and computer-controlled treatment machines. 3-DCRT enabled the delivery of more conformal dose distributions than hitherto possible, thus increasing the therapeutic ratio. 3-DCRT, when used in the treatment of early HCC, has been reported to provide local control rates of $71.4 \%$ to $93.8 \%$ and overall survival of $73.6 \%$ to $81.1 \%$ at 1 year. ${ }^{6,7}$ With the advent of intensity modulated radiotherapy (IMRT), the use of multiple fields and the modulation of the radiation dose intensity within the individual fields enables more conformal irradiation of irregularly shaped targets. The use of stereotactic principles and modern 3-DCRT, IMRT or volumetric arc therapy (VMAT) have further enabled the delivery of very high doses within the target volume with a sharp dose gradient outside the target volume, limiting dose to surrounding liver and normal tissues further. Daily image-guidance and respiratory motion management also characterize modern liver stereotactic body radiation therapy (SBRT) and are associated with the delivery of hypofractionated doses of radiation in a limited number of treatment sessions/fractions. The study of the tolerance of the normal liver and development of stereotactic techniques led to the adoption of SBRT as a technique of effectively treating liver tumors, although early reports generally included at least a subset of patients with advanced HCC. ${ }^{8,9}$ Subsequently, several institutions presented series of early HCC treated with high dose ablative radiation therapy, many including $\mathrm{HCC}>$ $5 \mathrm{~cm},{ }^{10}$ mostly these being patients ineligible for other ablative liver-directed therapies such as surgical resection, radiofrequency ablation (RFA) or microwave ablation (MWA).

The dosimetric advantage of using charged particle therapy to treat liver tumors was also explored by several institutions in Japan, Korea and the United States of America. Use of charged particles like protons or carbon ions instead of photons results in greatly reduced radiation dose deposited distal to the beam, enabling more conformal dose distributions and sparing more normal liver. The University of Loma Linda, ${ }^{11}$ the Massachusetts General Hospital $^{12}$ and the University of Tsukuba ${ }^{13}$ have all published their experiences with proton therapy as has a multiinstitutional Phase II study in the USA which reported 2-year local control of $94.8 \%$ in unresectable HCC. ${ }^{14}$ The Synchrony ${ }^{\mathrm{TM}}$ Respiratory Tracking System of the Cyberknife ${ }^{\circledR}$ Robotic Radiosurgery system is another platform for SBRT utilizing a linear accelerator mounted on a robotic arm to deliver multiple non-coplanar beam arrangements to the target while also tracking the tumor in real-time. Disadvantages of Cyberknife include the need for fiducial markers, especially to localize small early HCC. Magnetic resonance imaging (MRI) is also being explored to improve the accuracy of all steps in the process of delivering SBRT - from target delineation to treatment delivery as well as assessing response to SBRT. The use of MRI to guide target delineation is well established due to the greater clarity in depicting the extent of intrahepatic disease, as well as the information it 
provides regarding extent of motion of the liver/tumor. ${ }^{15}$ It is also used routinely in the clinic in assessing response post SBRT. ${ }^{16}$ The use of MRI in on-board image guidance during radiation delivery is being explored and expanded with the clinical availability of MR-Linear accelerators. ${ }^{17,18}$

This review proposes to summarize the existing literature for the role of SBRT in HCC, focusing on the role of ablative radiotherapy in treatment of $\mathrm{HCC}$, ie mostly tumors up to $3 \mathrm{~cm}$ in size. The radiobiological rationale behind the use of SBRT in early HCC is first described, followed by a review of existing evidence on the indications and outcomes of SBRT in this setting, highlighting studies that have compared SBRT to other treatments in early stage HCC patients. Further, controversies in the use of SBRT for early stage HCC are addressed as well as the future directions on this topic.

\section{Rationale Behind Use of SBRT in Early HCC}

SBRT refers to the use of hypofractionated doses of radiation in a limited number of sittings (typically less than or equal to 5) to ablate the tumor cells while causing minimal damage to surrounding normal liver through the use of highly conformal techniques.

The "5 R's of radiobiology", ie repair, reoxygenation, redistribution, repopulation and radiosensitivity are used to describe the mechanisms of cell response to conventionally fractionated external beam radiation, and the differential response of tumors versus normal tissues to radiation therapy is the basis for why radiation therapy has conventionally been delivered over many fractions. Techniques to focus radiation therapy with high precision and accuracy mean there is less need for prolonged fractionation. The effect of hypofractionated dose of radiation on tumor response, as typically used in SBRT, seemed to be supradditive to what could be explained by traditional radiobiological mechanisms. In SBRT, repair is hardly possible as the high dose delivered per fraction causes more necroptosis than apoptosis, therefore the majority of cells will suffer from lethal DNA damage rather than sublethal damage and die. Redistribution within the cell cycle is similarly rendered ineffective as the cell cycle is completely blocked at all stages after high dose irradiation - cells in both the sensitive and insensitive phases of the cell cycle are directly killed. ${ }^{19}$ While tumor hypoxia may persist after vascular injury due to SBRT, and reoxygenation may not be possible due to the relatively short duration of treatment, theoretically SBRT negates the effect of reoxygenation by causing direct cell kill of both normoxic and hypoxic tissues effectively due to the high doses delivered. SBRT also typically has shorter schedules than conventional fractionation, usually 1-2 weeks, thus giving cells no time to repopulate. Besides these mechanisms of direct cell kill, high dose SBRT has been shown in pre-clinical models to act through two additional mechanisms - vascular endothelial injury and immune cell activation. Endothelial apoptosis which is induced at single doses above $~ 8-11 \mathrm{~Gy}$, has anti-angiogenic effect in the tumor vasculature. ${ }^{20}$ Moreover, doses above 10 Gy induce vascular injury causing hypoxia, tumor microenvironment acidification and indirect death of tumor cells. ${ }^{21}$ Through increased vascular permeability and apoptosis, as well as endothelial injury induced platelet aggregation and thrombosis, SBRT further exacerbated hypoxia induced tumor necrosis. SBRT also recruits immune cells through activation of inflammatory cytokines such as IL-1 and TNF, resulting in an intense infiltrate of the tumor and distant sites by a cytotoxic CD-8+ cell infiltrate. Direct cell ablation by SBRT also releases tumor antigens profusely which are presented very effectively by these activated immune cells and further lead to immune cell activation and tumor cell kill, resulting in a vicious cycle of cell kill by immune mechanisms. ${ }^{22}$ Due to these direct cell-kill effects, SBRT can effectively ablate early HCC lesions and the parallel architecture of the functional subunits in the liver ensure that as long as a critical volume of normal liver is left unirradiated, the tumors can receive adequately ablative doses. In early HCC, the number of lesions is usually limited such that delivering ablative doses to these should be possible with negligible rates of toxicity, especially if the Child Pugh score is A or early B.

\section{Evidence for Indications and Efficacy of SBRT in Early HCC}

Traditionally, early stage HCC patients are treated with resection to provide cure in non-cirrhotic livers or in cirrhotic patients without portal hypertension, or with orthotopic liver transplant for selected early stage HCC patients, including those with impaired liver function. ${ }^{4}$ The use of locally ablative techniques such as radiofrequency ablation (RFA) is standard of care in cirrhotic livers. RFA in well-selected tumors $(<3 \mathrm{~cm}$ in size and technically accessible) results in high local control rates similar to 
surgical resection, ie more than $90 \%$ at $3-5$ years. However, only a minority of patients with early HCC are eligible for transplant (due to a limited donor pool), surgical resection or RFA due to tumor factors (size, number), liver function (Child Pugh score, portal hypertension), technical factors (location of tumor, proximity to large vessels) and general medical factors (coagulopathies, poor performance status). Hence there is a proportion of patients who require non-invasive ablative liver-directed therapies. These are currently the primary candidates who undergo external beam radiation using SBRT. Patients who have recurred following other local regional therapies are also candidates for SBRT.

There are 2 main indications for SBRT in early hepatocellular cancer - as definitive treatment, or as bridging treatment to orthotopic liver transplant. The target volumes and dose fractionation may be different when applied to each of these indications and the goals of care and outcomes of these two groups are different, as survival is largely driven by the use of liver transplantation in the latter category.

\section{Definitive Treatment of Early HCC}

Several institutions have reported their retrospective and prospective experiences with SBRT for early HCC's. Although many of the series have included a proportion of patients with advanced tumors, some have reported outcomes separately for early and advanced HCC. The early series from Korea and Japan mostly focus on treatment of early stage HCC which were ineligible for or failed other ablative therapies ${ }^{23-29}$ (Table 1). These series reported local control rates ranging from $67.5 \%$ to $97 \%$ at 3 years and overall survival rates of $39 \%$ to $84 \%$ at 3 years. Recent multi-institutional series from these countries though report outcomes of patients who were treated with SBRT upfront as first line of treatment, indicating a more widespread adoption/acceptance of SBRT in treatment of early tumors. ${ }^{30-37}$

\section{Bridging to Transplant}

Liver transplant is a curative treatment option for patients with $\mathrm{HCC}$ with background cirrhosis as it treats both the cancer as well as the cirrhosis. However, only patients with early stage HCC (eg within Milan criteria) are eligible for transplantation, and patients usually have to wait many months before an appropriate organ is available. In the interim, "bridging therapies" are used to prevent the tumor from progressing beyond transplant criteria. The most common bridging therapies include RFA/MWA and TACE, but SBRT has also been used effectively as a bridging therapy. ${ }^{38-40}$ SBRT has also been associated with pathological responses in the explanted livers ranging from $27 \%$ pathological complete responses to $73 \%$ pathological partial responses. ${ }^{41}$

This has been well tolerated with minimal toxicity and minimal drop-off from the waiting list. However, ultimately, orthotopic liver transplant drives the extremely good outcomes seen in this group.

\section{Comparison of SBRT with Other Techniques}

There are no large randomized controlled trials comparing SBRT to either surgical resection, RFA or TACE in early HCC. Below are summarized the available evidence (including some small RCT's and a recent non-inferiority Phase 3 RCT) comparing outcomes of SBRT with those of other liver-directed therapies such as RFA, TACE and surgical resection commonly used in early stage HCC.

\section{Radiofrequency Ablation (RFA)}

Wahl et al have reported a propensity-score matched analysis of patients treated with RFA or SBRT at a single institution and found that SBRT resulted in better local control in tumors larger than $2 \mathrm{~cm}^{42}$ (Table 2). Comparative analyses of outcomes and cost with SBRT and RFA for early HCC using the SEER database by Parikh et al concluded that there was no significant difference in survival, 90-day hospitalization, or costs of treatment with RFA vs SBRT. $^{43}$ Hara et al also conducted comparative propensity-score matched analyses between RFA and SBRT in small early HCC and reported similar excellent local control and survival in similarly selected patients. ${ }^{44}$ In contrast, Rajyaguru et al in a retrospective analysis from the National Cancer Database in patients with Stage I and II HCC treated with RFA or SBRT showed that RFA results in better overall survival; however several limitations of the data used for this analysis have been pointed out, including the lack of the baseline characteristics which are commonly used to decide patient selection for one of these two treatment modalities. ${ }^{45}$ More recently, a Markov model analysis compared the predicted OS of patients with small HCC $(<3 \mathrm{~cm},<3$ nodules, CP A or B with no vascular invasion) after RFA or SBRT. As per this modelling study, no significant difference in predicted overall survival was observed in both cohorts. 


\begin{tabular}{|c|c|c|c|c|c|c|}
\hline 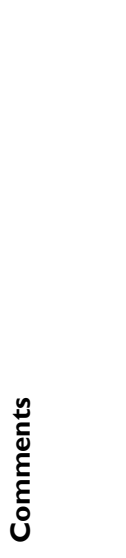 & 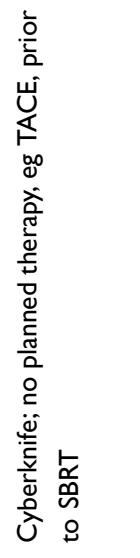 & 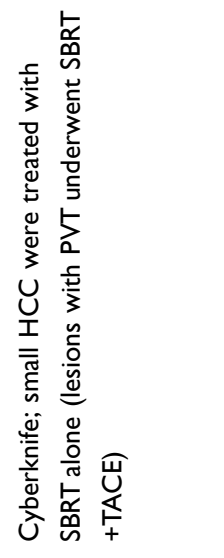 & 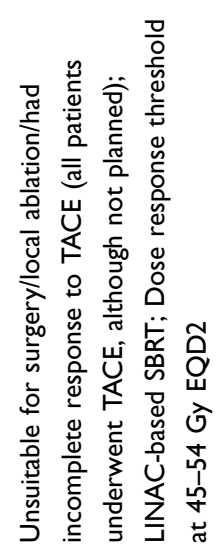 & 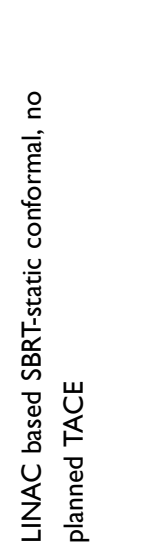 & 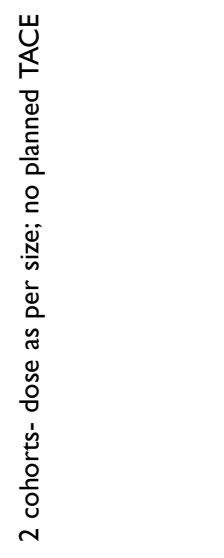 & 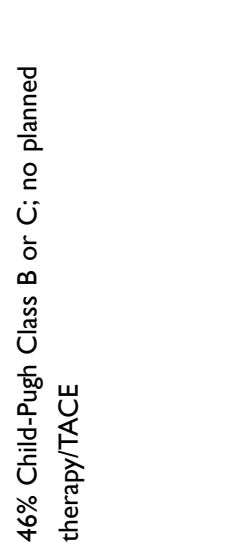 \\
\hline 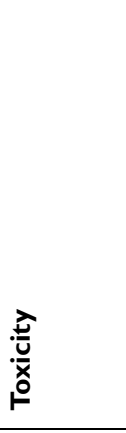 & 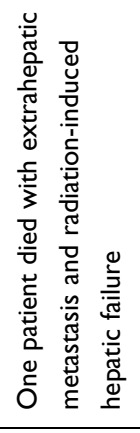 & 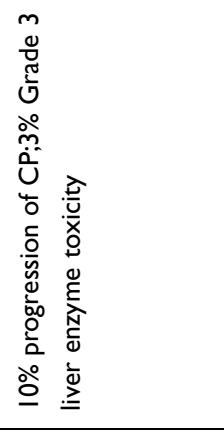 & 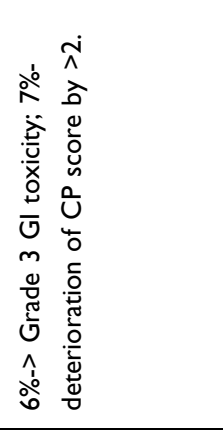 & 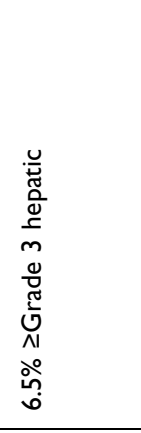 & 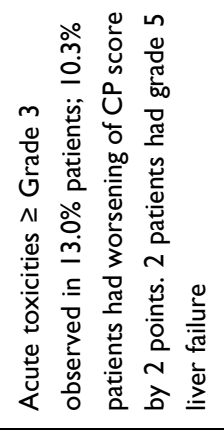 & 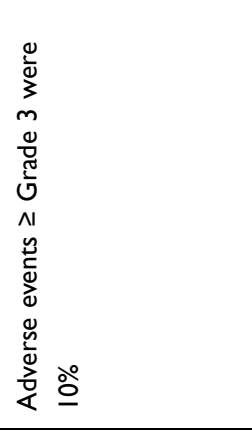 \\
\hline ถั & 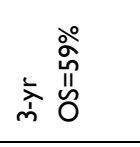 & 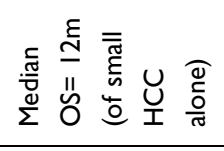 & 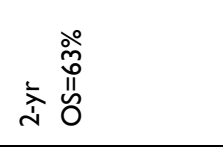 & 齐 & 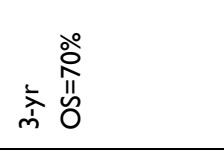 & 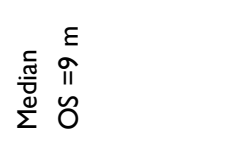 \\
\hline U & 离 & 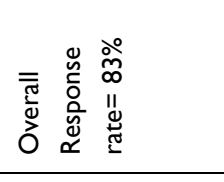 & 﨎 & 齐 & 产 & 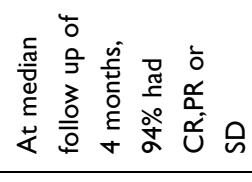 \\
\hline ڤั̀ & 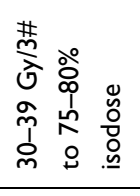 & 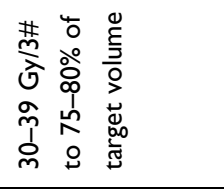 & 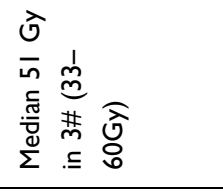 & 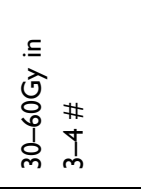 & 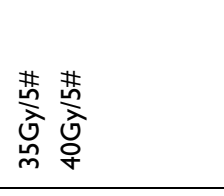 & 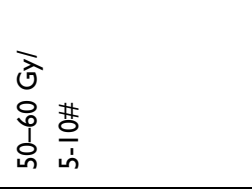 \\
\hline 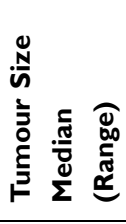 & 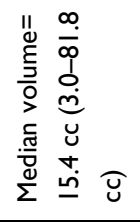 & 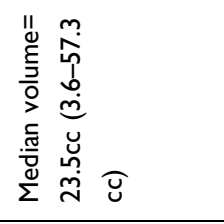 & 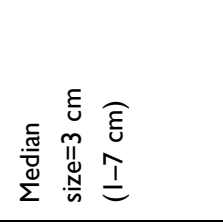 & 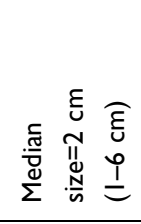 & 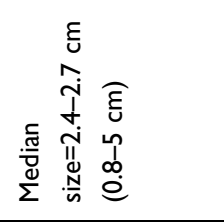 & 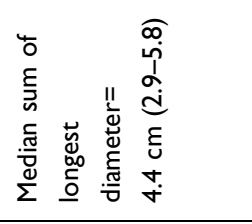 \\
\hline $\mathbf{z}$ & $\mathcal{f}$ & 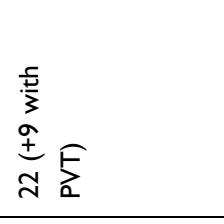 & 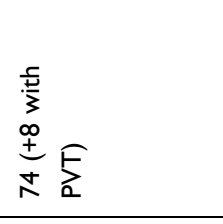 & a & $\stackrel{\stackrel{\llcorner}{\infty}}{-}$ & $\begin{array}{l}5 \\
\vdots \\
\circ \\
\stackrel{0}{0} \\
\infty \\
\dot{m} \\
\dot{\alpha}\end{array}$ \\
\hline ঠ્ & $\frac{0}{2}$ & 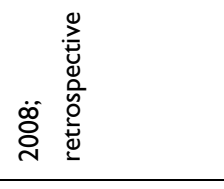 & 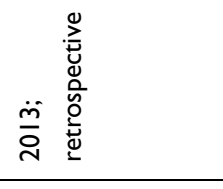 & $\frac{m}{i}$ & 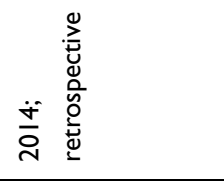 & 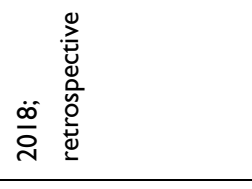 \\
\hline 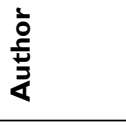 & 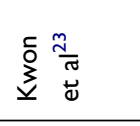 & $\begin{array}{l}\bar{\delta} \\
\bar{U} \\
\end{array}$ & 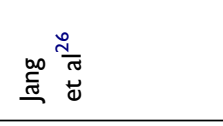 & 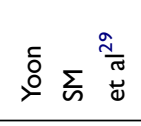 & 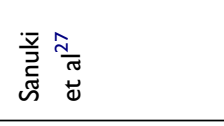 & $\stackrel{\frac{m}{0}}{\frac{m}{\sigma}}$ \\
\hline
\end{tabular}




\begin{tabular}{|c|c|c|c|c|c|c|c|c|}
\hline & 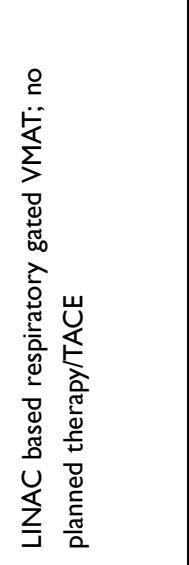 & 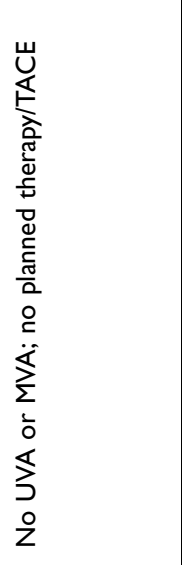 & 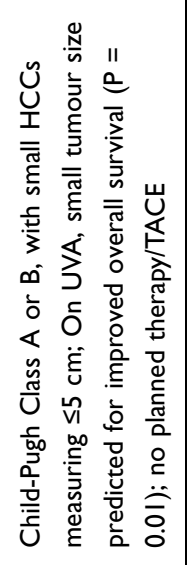 & 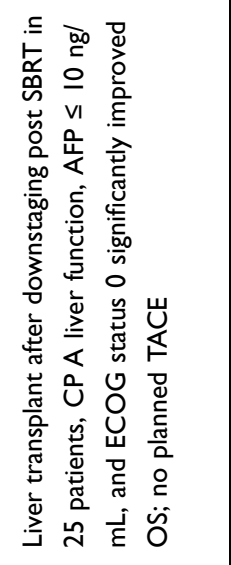 & 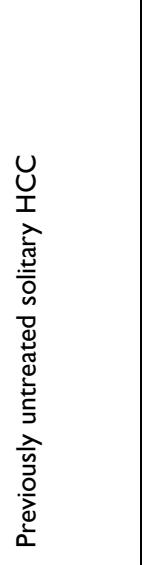 & 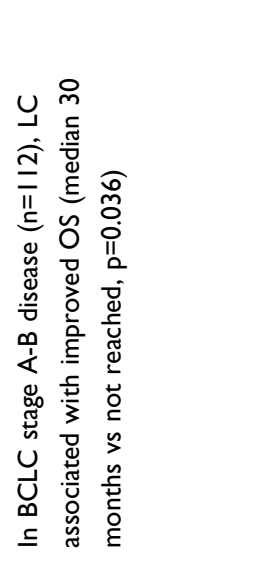 & 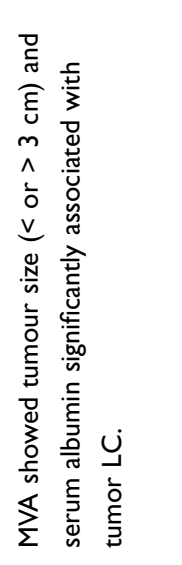 & \multirow{2}{*}{ 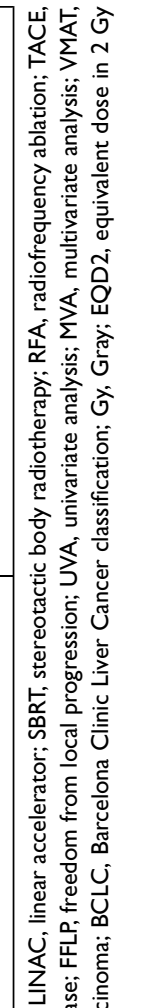 } \\
\hline 离 & 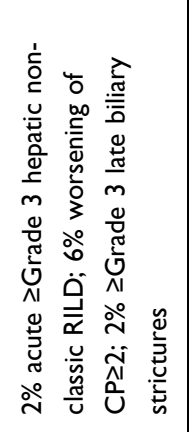 & 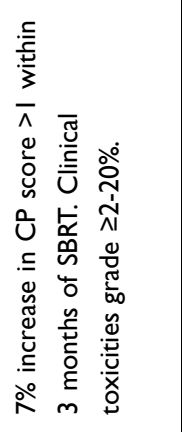 & 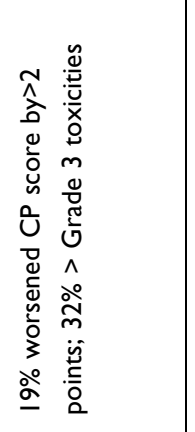 & 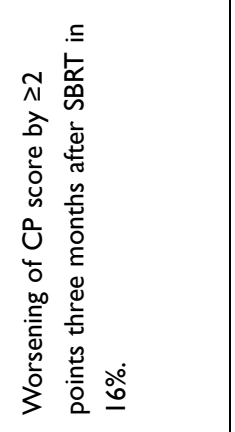 & 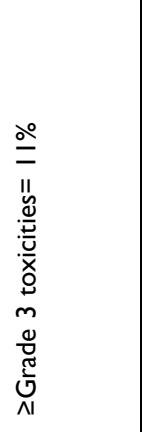 & 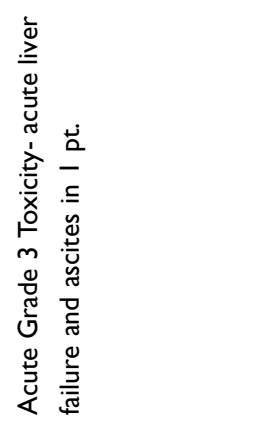 & 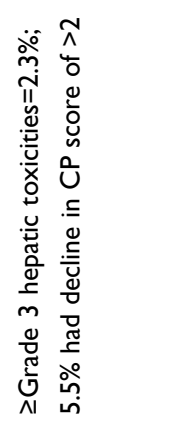 & \\
\hline ธั & 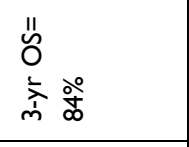 & 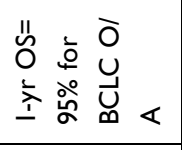 & 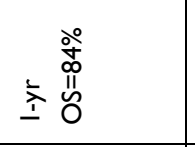 & 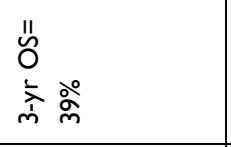 & 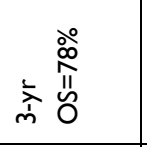 & 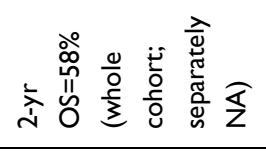 & 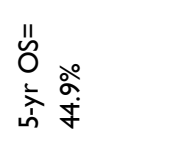 & 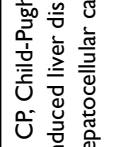 \\
\hline U & ले & 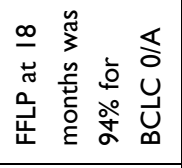 & 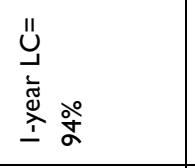 & 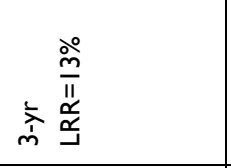 & 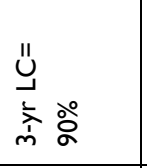 & 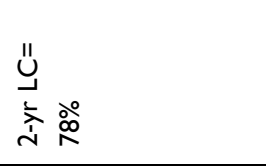 & 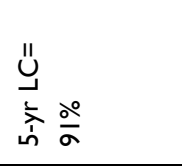 & \\
\hline ڤั & 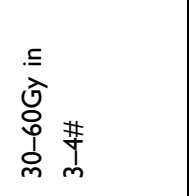 & 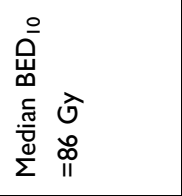 & 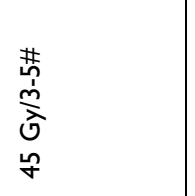 & 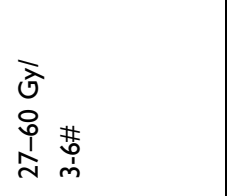 & $\begin{array}{l}\text { 葸 } \\
\text { 今 } \\
\text { o }\end{array}$ & 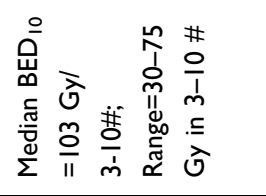 & 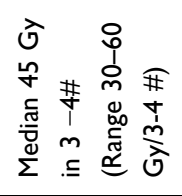 & 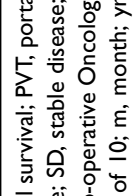 \\
\hline 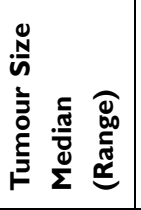 & 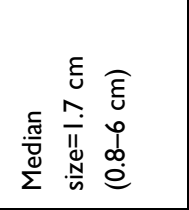 & 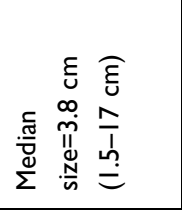 & 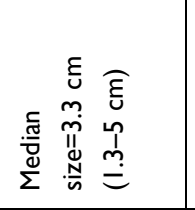 & 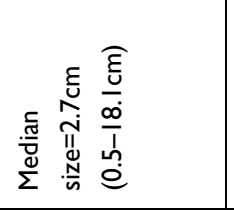 & 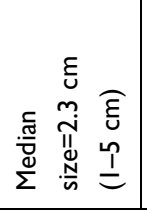 & 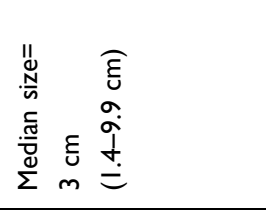 & 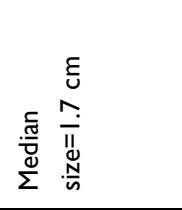 & \\
\hline $\mathbf{z}$ & $\stackrel{\varrho}{=}$ & 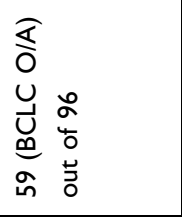 & $\bar{m}$ & 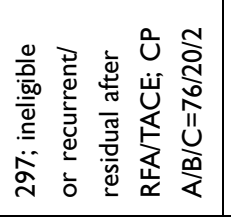 & 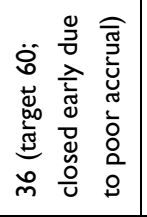 & 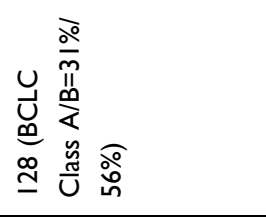 & 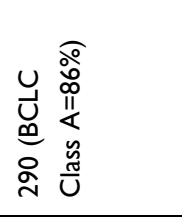 & \\
\hline 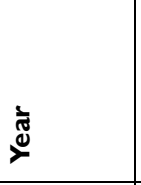 & 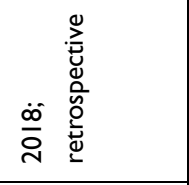 & 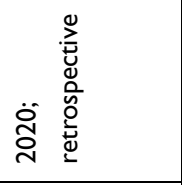 & 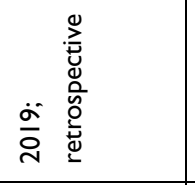 & 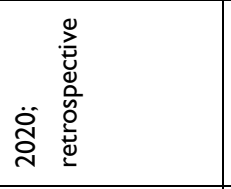 & 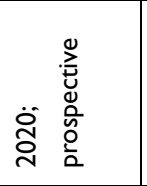 & 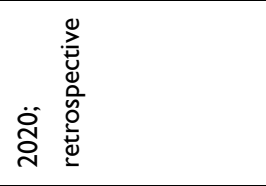 & 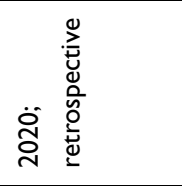 & \\
\hline $\begin{array}{l}\frac{1}{\partial} \\
\frac{c}{2} \\
\frac{1}{2}\end{array}$ & $\begin{array}{l}\infty \\
\stackrel{0}{0} \\
\stackrel{0}{\gamma}\end{array}$ & כ寻 & 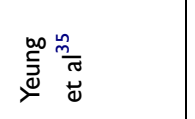 & 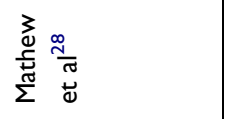 & 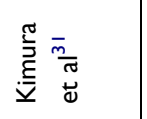 & $\bar{a} \frac{\tilde{n}}{\frac{\tilde{\sigma}}{\sigma}}$ & 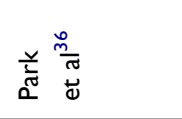 & \\
\hline
\end{tabular}




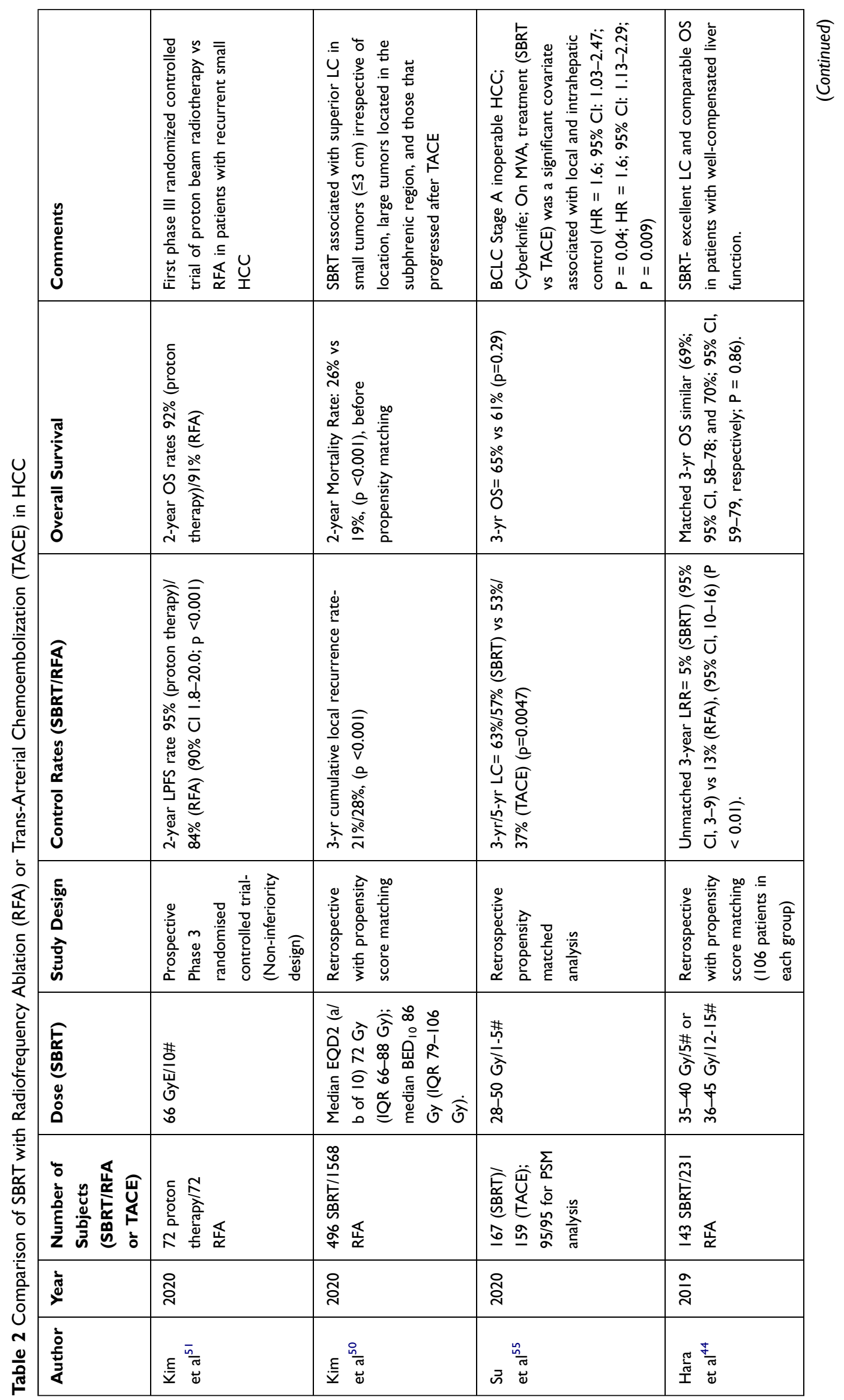




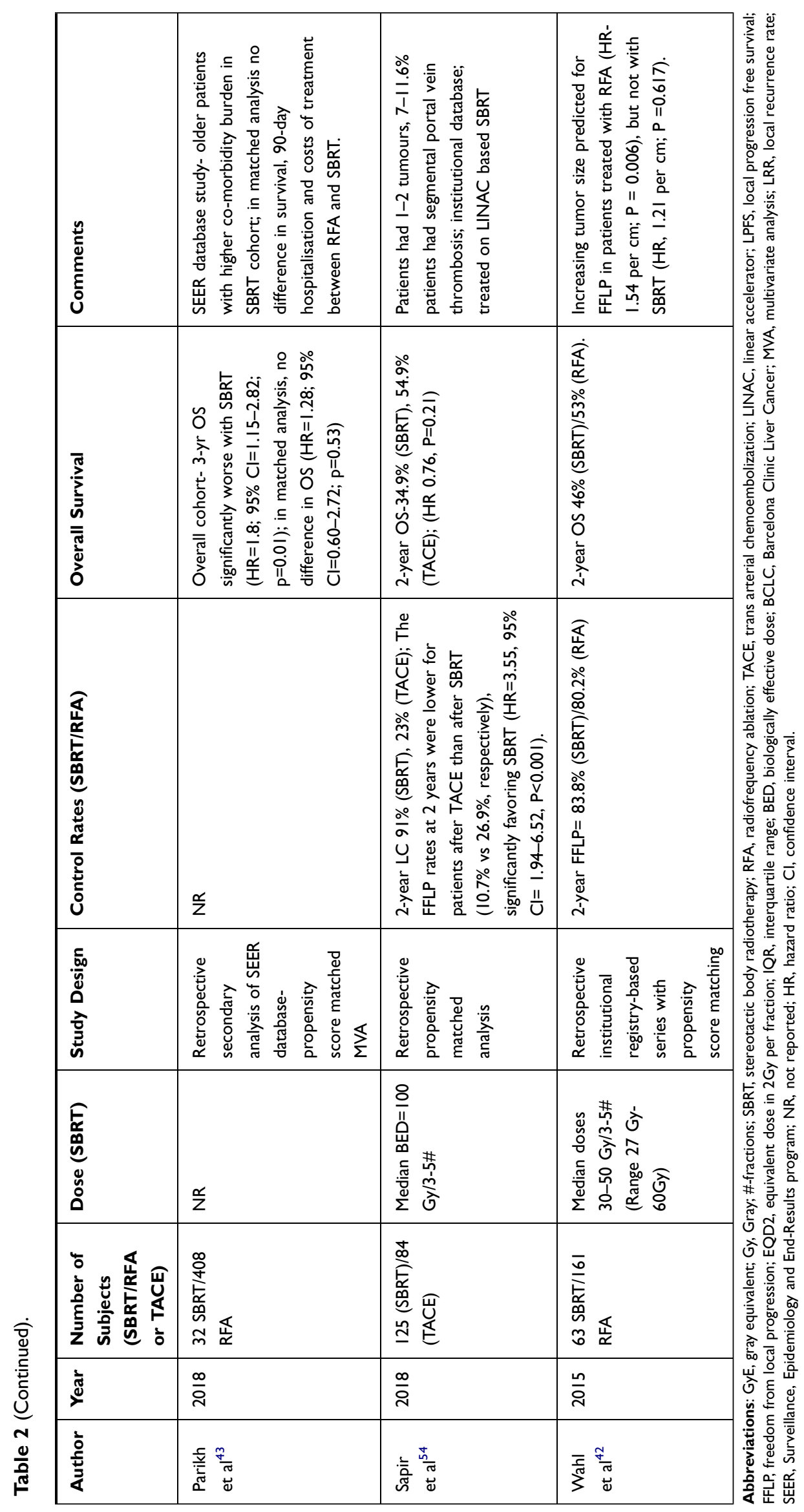


SBRT was found to result in better outcomes if tumor size was $2-3 \mathrm{~cm}^{46}$

Three large systematic reviews and meta-analyses have been reported recently comparing SBRT with RFA for early stage HCC, each of them including at least 2000 patients. Pan et al conducted a systematic review and meta-analysis of SBRT vs RFA in HCC, including 2783 patients and reported better local control of HCC using SBRT than RFA although overall survival was better with RFA. ${ }^{47}$ This was attributed to differences in the underlying liver function in the patients selected for each of these modalities. Similarly, in a separate review by Lee et al, SBRT and RFA had similar local control rates for small early HCC's but RFA showed a benefit as far as overall survival odds ratio was concerned when compared to SBRT. $^{48}$ On the contrary, Wang et al in a review and metaanalysis of 7928 patients, reported better local control of HCC with SBRT than with RFA especially for slightly larger tumors $(>2 \mathrm{~cm})$ with equivalent overall survival outcomes and comparable toxicity. ${ }^{49}$ In addition, a large multi-institutional retrospective study by Kim et al including 2064 patients reported significantly lower local recurrence rates with SBRT than with RFA in both the unmatched and propensity score matched cohorts. ${ }^{50}$ SBRT was also associated with superior local control in small tumors $\leq 3 \mathrm{~cm}$ irrespective of location, large tumors in subphrenic location and those that progressed after TACE.

The only randomized controlled trial comparing radiation therapy to RFA has been reported recently from the National Cancer Centre, Korea comparing proton beam therapy (PBT) to RFA in small recurrent/residual HCC, using a non-inferiority design. ${ }^{51}$ Patients with recurrent/residual HCC (size $<3 \mathrm{~cm}$, number $\leq 2$ ) were randomly assigned to receive $\mathrm{PBT}$ or RFA according to Child-Pugh score and tumor stage. The 2-year LPFS rate with PBT vs RFA was $92.8 \%$ vs $83.2 \%$, (90\% CI $0.7-$ $18.4 ; \mathrm{p}<0.001)$, meeting the criteria for non-inferiority. The 3- and 4-year LPFS rates for PBT were also noninferior to those for RFA. Four-year survival was $75-77 \%$, not significantly different in patients treated with either modality, when analyzed by intention to treat and per-protocol treatment. In this study, $26 \%$ of patients randomized to RFA crossed over to receive proton therapy, and $8 \%$ of patients randomized to proton therapy crossed over to receive RFA, demonstrating that factors such as tumor location and size make some tumors more favorable for RFA (eg smaller tumors, and away from large vessels) or SBRT (eg larger tumors, away from luminal GI tissues).

\section{Trans Arterial Chemoembolization (TACE)}

Although TACE is the recommended treatment of choice for intermediate stage BCLC tumors, BCLC also endorses the concept of treatment migration so that TACE is recommended for early stage HCC patients who have recurred or who are not amenable for the recommended standard treatments. Several prospective series have reported outcomes of TACE in such early HCC with median survivals ranging from 54.2 months, $^{52}$ to 3 -year survivals of $80.5 \%{ }^{53}$ A propensity-score matched analysis of patients treated with TACE or SBRT was reported by Sapir et al with overall survival at 1 and 2 years of $75.3 \%$ and $54.9 \%$, respectively, after TACE and $74.1 \%$ and $34.9 \%$ after SBRT, with no significant difference between treatment groups after adjustment for age, Child-Pugh score, baseline cirrhosis, liver transplantation, time from diagnosis of $\mathrm{HCC}$, and thrombosis of minor branches of PV (HR 0.76, $\mathrm{P}=0.21)^{54}$ (Table 2). A separate propensity-score matched analysis by $\mathrm{Su}$ et al in inoperable BCLC Stage A HCC also concluded that SBRT had better local control and intrahepatic control than TACE but showed comparable overall survival. $^{55}$ The accumulative 3- and 5-year OS rates were 65.1 , and $62.8 \%$ in the SBRT group and 61.0, and $50.4 \%$ in the TACE group, respectively $(\mathrm{P}=0.29)$. On multivariate analysis, treatment (SBRT vs TACE) was a significant covariate associated with local and intrahepatic control $(\mathrm{HR}=1.59 ; 95 \% \mathrm{CI}$ : 1.03-2.47; $\mathrm{P}=0.04$; $\mathrm{HR}=$ 1.61; 95\% CI: 1.13-2.29; P = 0.009). Some studies have also mentioned that combined treatment with SBRT +TACE was more effective than TACE alone. ${ }^{56} \mathrm{~A}$ recent preliminary analysis of a Phase III trial reported that use of SBRT after incomplete response to TACE/TAE as compared to further TACE/TAE itself resulted in significantly better local control (median not reached versus 8 months; $\mathrm{p}<0.0002$ ) in a single nodule, $5 \mathrm{~cm}$ or up to 3 nodules $<$ $3 \mathrm{~cm}$ in diameter. ${ }^{57}$ Other studies have reported similar control rates and survival in small HCC treated with SBRT alone or when combined with TACE also. ${ }^{58,59}$ The interim analysis of a randomized controlled trial from the University of Loma Linda comparing proton therapy to TACE in patients eligible for liver transplant also reported encouraging trend towards improvement in local control 
and $(88 \%$ vs $45 \%, p=0.06)$ and progression-free survival ( $48 \%$ vs $31 \%, p=0.06)$ favoring the proton beam treatment group. $25 \%$ of the proton patients who underwent liver transplant showed a pathological complete response as compared to $10 \%$ of those who received TACE. ${ }^{60}$

\section{Surgical Resection}

Although no head-to-head randomized trial has been reported between surgical resection and SBRT, in a propensity score matched series for patients with small primary HCC with 1 or 2 nodules and Child-Pugh A cirrhosis, surgical resection and SBRT performed similarly in terms of overall survival, local control and toxicity. ${ }^{61}$ However, in another study in patients with 1 to 3 small tumors ( $\leq 3 \mathrm{~cm}$ in diameter), surgical resection provided better long-term overall survival and disease-free survival. ${ }^{62}$ Further study of these two modalities may be facilitated among patients who are medically ineligible for surgery or those who refuse surgery.

\section{Future Directions}

There is ample scope for use of SBRT in early stage HCC, both in the definitive setting as well as in the setting of bridge to transplant and generation of higher level of evidence is essential. Randomized controlled trials comparing SBRT to current standards of care ie to other liverdirected therapies, in this patient group are much needed and, in some cases, already ongoing. ${ }^{63}$ Studies on clinically meaningful outcomes such as overall survival and progression-free survival, as well as cost-effectiveness of SBRT with regards to other liver-directed therapies are much needed. Another much less explored, but important, outcome is the quality of life of these patients who may struggle from symptoms of their HCC, underlying liver disease and/or treatment side effects, over a long time. ${ }^{64}$

The use of blood and imaging biomarkers in the selection of treatments for HCC is needed, beyond the use of Indocyanine green (ICG) dye clearance to assess liver function and guide dose schedules. ${ }^{65}$ More integration of radiological biomarkers, even the use of functional imaging to select patients who will benefit from SBRT needs further study.

\section{Disclosure}

Dr Laura A. Dawson reports grants from Raysearch, outside the submitted work. The authors report no other conflicts of interest in this work.

\section{References}

1. Blomgren H, Lax I, Näslund I, Svanström R. Stereotactic high dose fraction radiation therapy of extracranial tumors using an accelerator: clinical experience of the first thirty-one patients. Acta Oncol. 1995;34(6):861-870. doi:10.3109/02841869509127197

2. Dawson LA, Normolle D, Balter JM, McGinn CJ, Lawrence TS, Ten Haken RK. Analysis of radiation-induced liver disease using the Lyman NTCP model. Int J Radiat Oncol Biol Phys. 2002;53 (4):810-821. doi:10.1016/S0360-3016(02)02846-8

3. Sung H, Ferlay J, Siegel RL, et al. Global cancer statistics 2020: GLOBOCAN estimates of incidence and mortality worldwide for 36 cancers in 185 countries. CA Cancer J Clin. 2021;71:209-249. doi: $10.3322 /$ caac. 21660

4. European Association for the Study of the Liver; Galle PR, Forner A, Llovet JM, et al. EASL clinical practice guidelines: management of hepatocellular carcinoma. J Hepatol. 2018;69(1):182-236.

5. American Joint Committee on Cancer- Liver; Amin MB, Edge S, Greene F, et al. AJCC Cancer Staging Manual. 8th ed. New York: Springer; 2016.

6. Park HC, Seong J, Han KH, Chon CY, Moon YM, Suh CO. Doseresponse relationship in local radiotherapy for hepatocellular carcinoma. Int J Radiat Oncol Biol Phys. 2002;54(1):150-155. doi:10.1016/S0360-3016(02)02864-X

7. Lim DH, Lee H, Park HC, et al. The efficacy of high-dose 3-dimensional conformal radiation therapy in patients with small hepatocellular carcinoma not eligible for other local modalities. Am J Clin Oncol. 2013;36(2):162-166. doi:10.1097/ COC.0b013e3182438dae

8. Tse RV, Hawkins M, Lockwood G, et al. Phase I study of individualized stereotactic body radiotherapy for hepatocellular carcinoma and intrahepatic cholangiocarcinoma. J Clin Oncol. 2008;26(4):657-664. doi:10.1200/JCO.2007.14.3529

9. Mendez-Romero A, Wunderink W, Hussain SM, et al. Stereotactic body radiation therapy for primary and metastatic liver tumors: a single institution Phase I-II study. Acta Oncol. 2006;45 (7):831-837. doi:10.1080/02841860600897934

10. Andolino DL, Johnson CS, Maluccio M, et al. Stereotactic body radiotherapy for primary hepatocellular carcinoma. Int J Rad Oncol Biol Phys. 2011;81(4):e447-e453. doi:10.1016/j.ijrobp.2011.04.011

11. Bush DA, Kayali Z, Grove R, Slater JD. The safety and efficacy of high-dose proton beam radiotherapy for hepatocellular carcinoma: a Phase 2 prospective trial. Cancer. 2011;117(13):3053-3059. doi: $10.1002 /$ cncr.25809

12. Hong TS, DeLaney TF, Mamon HJ, et al. A prospective feasibility study of respiratory-gated proton beam therapy for liver tumors. Pract Radiat Oncol. 2014;4(5):316-322. doi:10.1016/j. prro.2013.10.002

13. Mizumoto M, Okumura T, Hashimoto T, et al. Proton beam therapy for hepatocellular carcinoma: a comparison of three treatment protocols. Int J Radiat Oncol Biol Phys. 2011;81(4):1039-1045. doi:10.1016/j.ijrobp.2010.07.015

14. Hong TS, Wo JY, Yeap BY, et al. Multi-institutional phase II study of high-dose hypofractionated proton beam therapy in patients with localized, unresectable hepatocellular carcinoma and intrahepatic cholangiocarcinoma. $J$ Clin Oncol. 2016;34(5):460-468. doi:10.1200/JCO.2015.64.2710

15. Dawson LA, Eccles C, Craig T. Individualized image guided iso-NTCP based liver cancer SBRT. Acta Oncol. 2006;45 (7):856-864. doi:10.1080/02841860600936369

16. Mendiratta-Lala M, Masch W, Shankar PR, et al. Magnetic resonance imaging evaluation of hepatocellular carcinoma treated with stereotactic body radiation therapy: long term imaging follow-up. Int J Radiat Oncol Biol Phys. 2019;103(1):169-179. doi:10.1016/j. ijrobp.2018.09.004 
17. Rosenberg SA, Henke LE, Shaverdian N, et al. A multi-institutional experience of MR-guided liver stereotactic body radiation therapy. Adv Radiat Oncol. 2018;4(1):142-149. doi:10.1016/j.adro.2018.08.005

18. Feldman AM, Modh A, Glide-Hurst C, Chetty IJ, Movsas B. Realtime magnetic resonance-guided liver stereotactic body radiation therapy: an institutional report using a magnetic resonance-linac system. Cureus. 2019;11(9):e5774. doi:10.7759/cureus.5774

19. Qiu B, Aili A, Xue L, Jiang P, Wang J. Advances in radiobiology of stereotactic ablative radiotherapy. Front Oncol. 2020;10:1165. doi:10.3389/fonc.2020.01165

20. Garcia-Barros M, Paris F, Cordon-Cardo C, et al. Tumor response to radiotherapy regulated by endothelial cell apoptosis. Science. 2003;300(5622):1155-1159. doi:10.1126/science.1082504

21. Park HJ, Griffin RJ, Hui S, Levitt SH, Song CW. Radiation-induced vascular damage in tumors: implications of vascular damage in ablative hypofractionated radiotherapy (SBRT and SRS). Radiat Res. 2012;177(3):311-327. doi:10.1667/RR2773.1

22. Bernstein M, Krishnan S, Hodge J, et al. Immunotherapy and stereotactic ablative radiotherapy (ISABR): a curative approach? Nat Rev Clin Oncol. 2016;13:516-524. doi:10.1038/nrclinonc.2016.30

23. Kwon JH, Bae SH, Kim JY, et al. Long-term effect of stereotactic body radiation therapy for primary hepatocellular carcinoma ineligible for local ablation therapy or surgical resection. Stereotactic radiotherapy for liver cancer. BMC Cancer. 2010;10:475. doi:10.1186/ 1471-2407-10-475

24. Choi BO, Jang HS, Kang KM, et al. Fractionated stereotactic radiotherapy in patients with primary hepatocellular carcinoma. Jpn J Clin Oncol. 2006;36(3):154-158. doi:10.1093/jjco/hyi236

25. Choi BO, Choi IB, Jang HS, et al. Stereotactic body radiation therapy with or without transarterial chemoembolization for patients with primary hepatocellular carcinoma: preliminary analysis. $B M C$ Cancer. 2008;8:351. doi:10.1186/1471-2407-8-351

26. Jang WI, Kim MS, Bae SH, et al. High-dose stereotactic body radiotherapy correlates increased local control and overall survival in patients with inoperable hepatocellular carcinoma. Radiat Oncol. 2013;8:250. doi:10.1186/1748-717X-8-250

27. Sanuki N, Takeda A, Oku Y, et al. Stereotactic body radiotherapy for small hepatocellular carcinoma: a retrospective outcome analysis in 185 patients. Acta Oncol. 2014;53(3):399-404. doi:10.3109/ 0284186X.2013.820342

28. Mathew AS, Atenafu EG, Owen D, et al. Long term outcomes of stereotactic body radiation therapy for hepatocellular carcinoma without macrovascular invasion. Eur J Cancer. 2020;134:41-51. doi:10.1016/j.ejca.2020.04.024

29. Yoon SM, Lim YS, Park MJ, et al. Stereotactic body radiation therapy as an alternative treatment for small hepatocellular carcinoma. PLoS One. 2013;8(11):e79854. doi:10.1371/journal.pone.0079854

30. Jeong Y, Jung J, Cho B, et al. Stereotactic body radiation therapy using a respiratory-gated volumetric-modulated arc therapy technique for small hepatocellular carcinoma. BMC Cancer. 2018;18(1):416. doi:10.1186/s12885-018-4340-7

31. Kimura T, Takeda A, Sanuki N, et al. Multicenter prospective study of stereotactic body radiotherapy for previously untreated solitary primary hepatocellular carcinoma: the STRSPH study. Hepatol Res. 2020. doi:10.1111/hepr.13595

32. Loi M, Comito T, Franzese C, et al. Stereotactic body radiotherapy in hepatocellular carcinoma: patient selection and predictors of outcome and toxicity. J Cancer Res Clin Oncol. 2020. doi:10.1007/s00432020-03389-2

33. Qiu H, Moravan MJ, Milano MT, Usuki KY, Katz AW. SBRT for hepatocellular carcinoma: 8-year experience from a regional transplant center. $J$ Gastrointest Cancer. 2018;49(4):463-469. doi:10.1007/s12029-017-9990-1
34. Liu HY, Lee Y, McLean K, et al. Efficacy and toxicity of stereotactic body radiotherapy for early to advanced stage hepatocellular carcinoma - initial experience from an Australian Liver Cancer Service. Clin Oncol. 2020;32(10):e194-e202. doi:10.1016/j. clon.2020.04.004

35. Yeung R, Beaton L, Rackley T, et al. Stereotactic body radiotherapy for small unresectable hepatocellular carcinomas. Clin Oncol. 2019;31(6):365-373. doi:10.1016/j.clon.2019.01.012

36. Park S, Jung J, Cho B, et al. Clinical outcomes of stereotactic body radiation therapy for small hepatocellular carcinoma. $J$ Gastroenterol Hepatol. 2020;35(11):1953-1959. doi:10.1111/jgh.15011

37. Sun J, Wang Q, Hong ZX, et al. Stereotactic body radiotherapy versus hepatic resection for hepatocellular carcinoma $(</=5 \mathrm{~cm})$ : a propensity score analysis. Hepatol Int. 2020;14:788-797. doi:10.1007/s12072-020-10088-0

38. Sapisochin G, Barry A, Doherty M, et al. Stereotactic body radiotherapy versus TACE or RFA as a bridge to transplant in patients with hepatocellular carcinoma. An intention-to-treat analysis. J Hepatol. 2017;67(1):92-99. doi:10.1016/j.jhep.2017.02.022

39. Moore A, Cohen-Naftaly M, Tobar A, et al. Stereotactic body radiation therapy (SBRT) for definitive treatment and as a bridge to liver transplantation in early stage inoperable Hepatocellular carcinoma. Radiat Oncol. 2017;12(1):163. doi:10.1186/s13014-017-0899-4

40. Mohamed M, Katz AW, Tejani MA, et al. Comparison of outcomes between SBRT, yttrium-90 radioembolization, transarterial chemoembolization, and radiofrequency ablation as bridge to transplant for hepatocellular carcinoma. Adv Radiat Oncol. 2015;1(1):35-42. doi:10.1016/j.adro.2015.12.003

41. O'Connor JK, Trotter J, Davis GL, Dempster J, Klintmalm GB, Goldstein RM. Long-term outcomes of stereotactic body radiation therapy in the treatment of hepatocellular cancer as a bridge to transplantation. Liver Transpl. 2012;18(8):949-954. doi:10.1002/1t.23439

42. Wahl DR, Stenmark MH, Tao Y, et al. Outcomes after stereotactic body radiotherapy or radiofrequency ablation for hepatocellular carcinoma. J Clin Oncol. 2016;34(5):452-459. doi:10.1200/ JCO.2015.61.4925

43. Parikh ND, Marshall VD, Green M, et al. Effectiveness and cost of radiofrequency ablation and stereotactic body radiotherapy for treatment of early-stage hepatocellular carcinoma: an analysis of SEER-medicare. $J$ Med Imaging Radiat Oncol. 2018;62 (5):673-681. doi:10.1111/1754-9485.12754

44. Hara K, Takeda A, Tsurugai Y, et al. Radiotherapy for hepatocellular carcinoma results in comparable survival to radiofrequency ablation: a propensity score analysis. Hepatology. 2019;69(6):2533-2545. doi:10.1002/hep.30591

45. Rajyaguru DJ, Borgert AJ, Smith AL, et al. Radiofrequency ablation versus stereotactic body radiotherapy for localized hepatocellular carcinoma in nonsurgically managed patients: analysis of the national cancer database. J Clin Oncol. 2018;36(6):600-608. doi:10.1200/ JCO.2017.75.3228

46. Seo YS, Kim MS, Yoo HJ, et al. Radiofrequency ablation versus stereotactic body radiotherapy for small hepatocellular carcinoma: a Markov model-based analysis. Cancer Med. 2016;5 (11):3094-3101. doi:10.1002/cam4.893

47. Pan YX, Fu YZ, Hu DD, et al. Stereotactic body radiotherapy vs. radiofrequency ablation in the treatment of hepatocellular carcinoma: a meta-analysis. Front Oncol. 2020;10:1639. doi:10.3389/ fonc. 2020.01639

48. Lee J, Shin IS, Yoon WS, Koom WS, Rim CH. Comparisons between radiofrequency ablation and stereotactic body radiotherapy for liver malignancies: meta-analyses and a systematic review. Radiother Oncol. 2020;145:63-70. doi:10.1016/j. radonc.2019.12.004 
49. Wang L, Ke Q, Huang Q, Shao L, Chen J, Wu J. Stereotactic body radiotherapy versus radiofrequency ablation for hepatocellular carcinoma: a systematic review and meta-analysis. Int J Hyperthermia. 2020;37(1):1313-1321. doi:10.1080/02656736.2020.1843719

50. Kim N, Cheng J, Jung I, et al. Stereotactic body radiotherapy versus Radiofrequency ablation in Asian patients with Hepatocellular carcinoma. $J$ Hepatol. 2020;73(1):121-129. doi:10.1016/j. jhep.2020.03.005

51. Kim TH, Koh YH, Kim BH, et al. Proton beam radiotherapy vs. radiofrequency ablation for recurrent hepatocellular carcinoma: a randomized phase III trial. J Hepatol. 2021;74(3):603-612. doi:10.1016/j.jhep.2020.09.026

52. Burrel M, Reig M, Forner A, et al. Survival of patients with hepatocellular carcinoma treated by transarterial chemoembolisation (TACE) using Drug Eluting Beads. Implications for clinical practice and trial design. $J$ Hepatol. 2012;56(6):1330-1335. doi:10.1016/j. jhep.2012.01.008

53. Bargellini I, Sacco R, Bozzi E, et al. Transarterial chemoembolization in very early and early-stage hepatocellular carcinoma patients excluded from curative treatment: a prospective cohort study. Eur J Radiol. 2012;81(6):1173-1178. doi:10.1016/j.ejrad.2011.03.046

54. Sapir E, Tao Y, Schipper MJ, et al. Stereotactic body radiation therapy as an alternative to transarterial chemoembolization for hepatocellular carcinoma. Int J Radiat Oncol Biol Phys. 2018;100 (1):122-130. doi:10.1016/j.ijrobp.2017.09.001

55. Su TS, Liang P, Zhou Y, et al. Stereotactic body radiation therapy vs. transarterial chemoembolization in inoperable barcelona clinic liver cancer stage a hepatocellular carcinoma: a retrospective, propensity-matched analysis. Front Oncol. 2020;10:347. doi:10.3389/fonc. 2020.00347

56. Jun BG, Kim SG, Kim YD, et al. Combined therapy of transarterial chemoembolization and stereotactic body radiation therapy versus transarterial chemoembolization for $\leq 5 \mathrm{~cm}$ hepatocellular carcinoma: propensity score matching analysis. PLoS One. 2018;13(10): e0206381. doi:10.1371/journal.pone.0206381

57. Comito T, Loi M, Franzese C et al. SBRT vs TAE/TACE in hepatocellular carcinoma: results from a Phase III trial (NCT02323360). ePoster Abstract (PB02-02) presented at: Liver Cancer Summit of the European Association for Study of Liver; February 6-8; 2020; Prague, Czech Republic.
58. Kimura T, Aikata H, Doi Y, et al. Comparison of stereotactic body radiation therapy combined with or without transcatheter arterial chemoembolization for patients with small hepatocellular carcinoma ineligible for resection or ablation therapies. Technol Cancer Res Treat. 2018;17:1533033818783450. doi:10.1177/1533033818783450

59. Wong TC, Chiang CL, Lee AS, et al. Better survival after stereotactic body radiation therapy following transarterial chemoembolization in nonresectable hepatocellular carcinoma: a propensity score matched analysis. Surg Oncol. 2019;28:228-323. doi:10.1016/j.suronc.2019.01.006

60. Bush DA, Smith JC, Slater JD, et al. Randomized clinical trial comparing proton beam radiation therapy with transarterial chemoembolization for hepatocellular carcinoma: results of an interim analysis. Int $J$ Radiat Oncol Biol Phys. 2016;95(1):477-482. doi:10.1016/j.ijrobp.2016.02.027

61. Su TS, Liang P, Liang J, et al. Long-term survival analysis of stereotactic ablative radiotherapy versus liver resection for small hepatocellular carcinoma. Int J Radiat Oncol Biol Phys. 2017;98 (3):639-646. doi:10.1016/j.ijrobp.2017.02.095

62. Nakano R, Ohira M, Kobayashi T, et al. Hepatectomy versus stereotactic body radiotherapy for primary early hepatocellular carcinoma: a propensity-matched analysis in a single institution. Surgery. 2018;164(2):219-226. doi:10.1016/j.surg.2018.03.006

63. ClinicalTrials.gov. Bethesda (MD): National Library of Medicine (US) August 6, 2019- identifier NCT04047173, Radiofrequency Ablation (RFA) versus Stereotactic Body Radiotherapy (SBRT) for the treatment of recurrent small hepatocellular carcinoma: a prospective, open, randomized, controlled clinical study; 2020. Available from: https:/clinicaltrials.gov/ct2/show/NCT04047173? term=NCT04047173\&draw=2\&rank=1. Accessed May 11, 2021.

64. Mutsaers A, Greenspoon J, Walker-Dilks C, Swaminath A. Systematic review of patient reported quality of life following stereotactic ablative radiotherapy for primary and metastatic liver cancer. Radiat Oncol. 2017;12(1):110. doi:10.1186/s13014-017-0818-8

65. El Naqa I, Johansson A, Owen D, et al. Modeling of normal tissue complications using imaging and biomarkers after radiation therapy for hepatocellular carcinoma. Int J Radiat Oncol Biol Phys. 2018;100 (2):335-343. doi:10.1016/j.ijrobp.2017.10.005
Journal of Hepatocellular Carcinoma

\section{Publish your work in this journal}

The Journal of Hepatocellular Carcinoma is an international, peerreviewed, open access journal that offers a platform for the dissemination and study of clinical, translational and basic research findings in this rapidly developing field. Development in areas including, but not limited to, epidemiology, vaccination, hepatitis therapy, pathology and molecular tumor classification and prognostication are all considered for publication. The manuscript management system is completely online and includes a very quick and fair peer-review system, which is all easy to use. Visit http://www.dovepress.com/ testimonials.php to read real quotes from published authors. 